\title{
Research on Pervasive Knowledge Service Field
}

\author{
Min YAO ${ }^{1, \text { a }}$, Zhongwei LIU ${ }^{1, b}$, Yao YAO ${ }^{2, c}$ \\ ${ }^{1}$ College of Computer Science and Technology, Zhejiang University, Hangzhou, China \\ ${ }^{2}$ Hangzhou Institute of Service Engineering, Hangzhou Normal University, Hangzhou, China \\ aemail: myao@zju.edu.cn, bemail:derrenang@foxmail.com, email: yyaoexist@163.com
}

\begin{abstract}
Keywords: Knowledge service; pervasive knowledge service field; ecology characteristics
\end{abstract}
\begin{abstract}
Pervasive knowledge service is the service to people-oriented. Drawing lessons from the basic theory about field in physics and based on pervasive knowledge service space (PKS ${ }^{2}$ ), the paper constructs the model of pervasive knowledge service field by means of multidisciplinary research achievements such as intelligence computing, pervasive computing, ecology, system science, knowledge science and service science, and discusses the ecology characteristics of pervasive knowledge service field. At the same time, the key technologies of pervasive knowledge service in design and implementation are discussed as well.
\end{abstract}

\section{Introduction}

Pervasive knowledge service (PKS) is a kind of service which introduces the concept of "people-oriented" in pervasive computing to knowledge service and puts focus on providing problem-oriented or task-oriented knowledge solutions to users. It enables knowledge service to achieve pervasiveness, mobility, transparency, quality control, personality, self-adaptability, initiative, security, easy-to-use and agility. Pervasive knowledge services may be accessed seamlessly through any format at any time and any place.

Pervasive knowledge service (PKS) is a quaternion [1], that is, PKS $=(\mathrm{P}, \mathrm{K}, \mathrm{S}, \mathrm{T})$. Where:

(1) P is the main body of PKS, i.e. human, and it is the combination of producer, consumer and decomposer for knowledge;

(2) $\mathrm{K}$ is the set of knowledge, including various facts, principles, paradigms and so on;

(3) $\mathrm{S}$ is the set of service, including various service manners and service techniques;

(4) $\mathrm{T}$ is the time witnessing the evolution processes of knowledge and the evolution chapters of service technology.

PKS can be visualized by pervasive knowledge service space. Pervasive knowledge service space $\left(\mathrm{PKS}^{2}\right)$ is a three dimensional space which takes human as origin and takes knowledge, service and time as coordinates. Here, human $(\mathrm{P})$ is the origin of pervasive knowledge service space reflecting the fundamental properties of people-oriented and people-oriented. The knowledge service plane which is called Pervasive Knowledge Service Field (short by PKSF) formed by knowledge dimension (K) and service dimension (S) reflects that knowledge service is the fusion of knowledge and service.

Drawing on the experience of basic theory of field in physics, we constructs the model of pervasive knowledge service field by means of multidisciplinary research achievements such as intelligence computing, pervasive computing, ecology, system science, knowledge science and service science and discusses the ecology characteristics of pervasive knowledge service field. At the same time, the key technologies in design and implement of pervasive knowledge service are discussed as well.

\section{Pervasive Knowledge Service Field Mode}

Pervasive knowledge service field is a virtual space rather than a physical space.

Definition 1: Pervasive knowledge service field (PKSF) is a quaternion,

$$
\operatorname{PKSF}=\left(P_{\Sigma}, F_{p}, F_{s}, t\right)
$$


Here, $P_{\Sigma}$ is the set of particles which consist PKSF, $F_{p}$ is the forces between particles, $F_{S}$ is the strength of particles, $A_{s}$ is the construction of PKSF, the following definition will make the concrete meaning clear; $t$ represents time which reflects the attribute of changing over time.

Definition 2: There are 3 kinds of basic particles in pervasive knowledge service field:

$P_{\Sigma}=(P, K, S)$

Where:

(1) $P$ which represents human is the most active particle with subjective initiative and is used for creating, manipulating and disseminating knowledge.

(2) $K$ is knowledge particle. It is the basic indivisible knowledge unit. $K$ could be the knowledge module which describes principles, facts, common senses or paradigms; it also could be the knowledge web pages.

(3) $S$ is the particle of service. It is the basic indivisible service unit. $S$ could be relevant service module; it also could be service web pages.

Definition 3: The force between 2 particles in PKSF is defined as:

$$
F_{p}=k \frac{S_{p}}{D_{p}}
$$

Where

(1) $k$ is ratio constant;

(2) $S_{p}$ is the assimilation measure between two particles;

(3) $D_{p}$ is the dissimilation measure between two particles.

It should be explained that assimilation measure can be confirmed by the genre between two particles, that is to say, assimilation measure could be one of the many genres such as matching degree, similarity degree, relevance degree or satisfied degree between particles. In a similar way, Dissimilation measure could be one of the many genres such as mismatching degree, diversity degree, dissimilarity degree, irrelevance degree or dissatisfy degree between particles.

Definition 4: In pervasive knowledge service field, the ratio of the force that acts on particles to the number of particles is called particles intensity $F_{S}$,

$$
F_{S}=\frac{\sum_{i} F_{i}}{Q}
$$

Here, $Q$ is the volume of the particle, $F_{i}$ is the force between this particle and the number i particle.

Definition 5: Pervasive knowledge service field is a dynamic non-linear ecological evolutionary system. It consists of 3 kinds of basic particles and its construction $\left(A_{s}\right)$ have features below:

(1) Particles in PKSF can combine together to form compound particle.

(2) Particles and compound particles can combine together as required to form group. One particle can appear in different groups at the same time. It is to say that to particles, the construction of group is overlapped.

(3) Pervasive knowledge service field can be divided into some sub-fields based on domains or assignments. In a similar way, to particles or groups, the construction of sub-field is overlapped.

(4) With the passing of time $t$, knowledge service field can always form the grand evolutionary chapter along with dynamic evolution, equilibrium evolution and harmonious evolution interlaced with each other.

Theorem 1: Energy growth law With the development of society and the progress of technology, especially with the development of internet and internet of thing, the energy of PKSF has been increasing all the time. It does not obey the law of conservation of energy in traditional physics.

Definition 6: PKSD is a radiation plane taking particle $P$ as origin/center, knowledge $(K)$ and service $(S)$ as coordinates, shown in figure 1.

It is necessary to note that different from the axis in Cartesian coordinate system, the knowledge 
coordinate and the service coordinate radiate from the origin which is concerned as center. Particles on knowledge coordinate and the service coordinate are knowledge particles and service particles; particles in other position are the compound particles consisting of knowledge particles and service particles and they are called knowledge service particles $K S$.

Definition 7: Let $d$ be the distance between the origin and the service particle $K S_{x}$ located in PKSF plane at point $x, K S_{x}$ meets the relation that the current requirement is inversely proportional to the distance $d$,

$$
M_{s}=\frac{c}{d}
$$

Here, $M_{s}$ is the satisfaction degree of particle $K S_{x}$ towards particle $P, c$ is a constant properly selected.

Obviously, in PKSF plane, knowledge particles, service particles and knowledge service particles are located in the plane by demand satisfaction degree towards particle $P$. The closer to the origin point, the more demand satisfaction towards particle $P$ the particle will get.

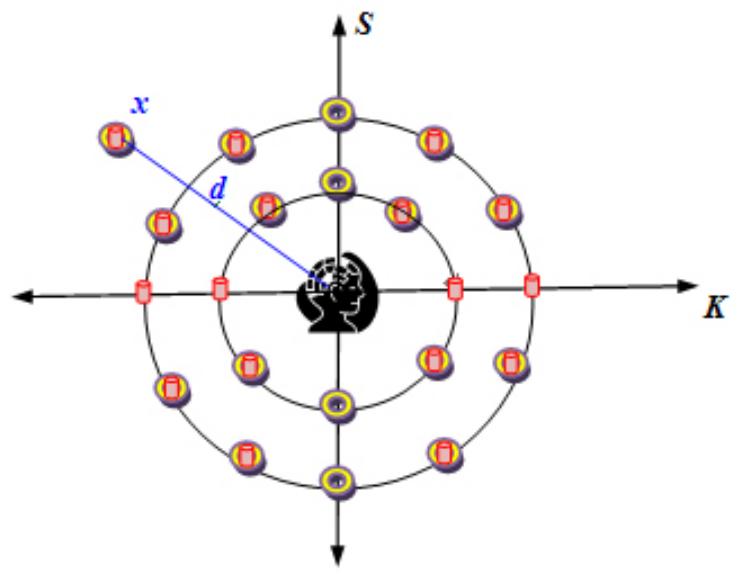

Fig.1. 2D Expression of PKSF

Definition 8: In PKSF plane, drawing a series of concentric circles with origin point to be the centre and $r_{i}=(i=1, \cdots, n)$ to be the radius, if all particles located on the same circumference have same demand satisfaction degree, then the concentric circle in PKSF plane will be the circle of equivalence demand satisfaction degree.

Theorem 2: The radiation plane appeared in figure 1 has the features below:

(1) Different particles Ps have different requirements, so as the radiation planes. It is to say that radiation planes differ from man to man.

(2) One particle $P$ has different demands at different time, so as the radiation planes. It is to say that radiation planes differ from time to time.

(3) Different particles Ps can be associated with each other through knowledge particles and service particles. Radiation planes which link the particles Ps compose the complicated dynamic evolutionary radiation plane group.

\section{Ecological Characteristics of PKSF}

As mentioned in literature [1], knowledge particles in pervasive knowledge service field has the ecological attributes like collectivity, relativity, purposefulness, sharing and so on; service particles has the ecological attributes of interaction, diversity and individualization. In this paper, we emphases discussing the environmental suitability and ecological balance in knowledge service field.

\subsection{Environmental Suitability}

The environmental suitability of knowledge service field is the changes of system trait about the relations between different parts inside the system and the functions in the system to adapt to new 
social demands and new social problems by means of reconstruction, learning and so on. It is better to express the concept of people-oriented.

(1) The adaptability of particle $K$

The adaptability of knowledge particle $K$ includes two parts below:

a) The updating process of particle content to adapt to the demands of solving new problems;

b) The adjusting process of particle construction to adapt to new requirements.

Especially, it is necessary to note that, in PKSF, the adaptability of knowledge particle $K$ is always evolving by way of better understanding.

(2) The adaptability of particle $S$

The adaptability of service particle $S$ mainly reflects in timely adaptation of the service particle $S$ 's construction and function to the changes of knowledge particle $K$.

(3) The adaptability of particle $P$

As mentioned before, particle $P$ is the most active particle with subjective initiative. The role of particle $P$ is to producing, using and disseminating knowledge. The adaptability of particle $P$ will be reflected by following processes: analysis of new problems and environment, self-adapting learning, new knowledge achievement, design of new knowledge particle $K$ and service particle $S$ and constructing new service algorithm.

Environmental suitability commonly exists. The environmental suitability of PKSF forms the morphological structure and the inside trait of behavior adapting to environment by way of interactive dynamic evolution.

\subsection{Ecological Balance}

The ecological balance of knowledge service field is relative and dynamic balanced. Such ecological equilibrium meets the rules of interacting and interdependence, information circulation and transformation, dynamic equilibrium, co-evolution and terminal threshold.

(1) The rule of interacting and interdependence

In PKSF, three basic kinds of particles and compound particles can combine as groups by rules; some groups can combine as sub-fields by rules. Every group or sub-field usually occupies a certain area, so that it forms an ecological sub-system with certain attributes. In one sub-system, not only particles or groups in same kind, but also particles or groups in different kinds have the relationship of interaction and interdependence; there are relationships of interaction and interdependence among different sub-systems and it is also can be described as having influences over each other. Some of such influences are direct, some are indirect; and some are instantly showed up, some are showed up after period of time. Therefore, during the constructing of PKSF, it is necessary to recognize the relationships among particles or groups. There is a need to make a reasonable and overall arrangement while structuring the PKSF.

(2) The rule of information circulation and transformation

All things consist of conflict. Both sides of conflict are interdependent, associated, included and completely understood with each other, and at the same time, realizing mutual transformation under certain conditions. Obviously, particles which compose PKSF with the strength of realize mutual attraction under the force of and mutual transformation with certain rules. For instance, when particle $P$ provides knowledge or service to other particles, the role of $P$ will become knowledge particle or service particle.

(3) The rule of dynamic equilibrium

Dynamic equilibrium reflects the rule of gross change inside the things while individual should obey the rule of moving balance. The dynamic equilibrium of PKSF is relative rather than absolute; moving rather than still; changed rather than unchanged. It what is the result of all individuals' consolidated movements adjusts itself from inside. It must be noted that the dynamic equilibrium of PKSF reflects the internal discipline which means there are relative balanced relationship among every particles or groups.

(4) The rule of co-evolution

Co-evolution means that the evolution processes of particles in the same sub-fields or groups in PKSF have the relationship of being associated with each other and gaining profit from others. It 
has two meanings: One means the direct benefits gaining from others among sub-fields and particle individuals; the other one means the interacting effect among different kinds of sub-fields or groups for the purpose of gaining ecological balance. Co-evolution advocates the achievement of global stable state in the field and the work division as well as cooperation used for maintaining such stable state.

\section{Key Technology}

According to the discussion of knowledge service field and knowledge service field ecological characteristics above, aspects listed below in pervasive knowledge service field should be primarily studied:

(1) The architecture of PKSF

According to the analysis of the characteristics which PKSF has and the characteristics of the demands for acquiring knowledge resources as well as service resources,

(2) Knowledge organization

Knowledge is the abstract part after the information processing. The knowledge organization in PKSF must reflect the concept of "people-oriented" and the ecological attributes of knowledge resources such as collectivity, relativity, purposefulness and sharing.

(3) Service organization

Service is the core issue of PKSF. The service organization in PKSF must reflect the ecological attributes of service resources such as interaction, diversity and individualization.

(4) The association between knowledge and service

Designing the technology of incidence mapping, ontology mapping, semantic mapping, fuzzy mapping and affinity mapping, It can implement the reasonable association between knowledge and service, so that to provide the service with high quality to the users rapidly and conveniently.

According to the requirements of PKSF mentioned above, the key technology of knowledge service field can be summarized as follow:

(1) Architecture of PKSF

Architecture and performance of knowledge service field are the key facts to implement pervasive knowledge service [2-6]. While designing the architecture, to increase the efficiency, the response speed and the adaptability of system maximally, and to lower the complexity of system, Service Oriented Architecture (known as SOA) will be referenced, the demand-driven service and the service-driven technology will be carried out and loosely-coupled application components such as service components, paradigm components or configuration components will be deployed, combined and manipulated by way of distribution according to the demands.

(2) Technology of knowledge organization

Pervasive knowledge service is a service emphasizes the concept of "people-oriented", therefore, the way to construct knowledge should centre on "people". It is to say that knowledge should be organized by way which can be easily understood by people, rather than the way of traditional engineering $[7,8]$. In order to form the library of components or paradigms which are required by knowledge service assignments, it is necessary to use semantic grid, otology technology, fuzzy language technology and intelligent analysis technology comprehensively to build the problem oriented or task oriented knowledge components or problem solving paradigms. And at the same time, exhibit the process of knowledge inference by way of visualization to facilitate users' understanding and identification.

(3) Technology of service organization

Services in PKSF include automatic knowledge service provided by internet system and services provided by all kinds of knowledge organizations. Along with the popularization of Internet and the development of Internet of thing, network service has already been main trend [9-12]. Network service has changed from simply sending or sharing message to comprehensive, multidimensional and multifunctional complex service system. Network service grows continuously with network as carrier and technology as means and emerges a scene of prosperity.

Accordingly, automatic knowledge service provided by intelligent network system is the 
emphasis to discuss. To show the interaction, diversity and individualization attributes of service resources, it is of great significant to use multiple technologies such as semantic technology, intelligent computing technology (like fuzzy technology, artificial neural networks and evolution computing etc.) and the inference technology based on paradigms to build fast and efficient knowledge service components.

(4) Technology of the association between knowledge and service

Using otology technology, semantic technology and biological intelligence technology to design problem-oriented or task-oriented multi-mapping such as association mapping, otology mapping, semantic mapping, fuzzy mapping and affinity mapping between knowledge and service, and build an efficiency paradigms library for problem solving.

It should be noticed that with the popularization of Internet and the development of cloud computing, pervasive knowledge service shall have the "cloud" features. That means PKSF consists of "knowledge cloud" and "service cloud". So, it is necessary to discuss the construction and the evolution appeared in PKSF under cloud environment.

\section{Conclusions}

This paper has brought forward the basic concepts of pervasive knowledge service field borrowing ideas from the concept of "field" in physics; formed pervasive knowledge service field model; discussed the ecological characteristics of pervasive knowledge service field; and at the same time, constructed the frame of pervasive knowledge service, designed knowledge particle, service particle and compound particle and developed the pervasive knowledge service system.

\section{Acknowledgement}

In this paper, the research was sponsored by National Support Program (No. 2014BA D10B02).

\section{References}

[1] Min Yao, Xinhe Wu, Yao Yao, Yiwen Wang, Research on Pervasive Knowledge Service Model, IEEE IEEM 2010 [C], 906 - 910

[2] F Kong, J Zuo, M Wu, Resource Classification and Service Matching of the Mold Manufacturing Grid Service Platform [J], Advances in Intelligent and Soft Computing, 2012, Vol. 114, pp. 757-764

[3] Yan-wen Wu, Qi Luo, Yu-jun Liu, Ying Yu, Zhao-hua Zhang. Research on Personalized Knowledge Service System in Community E-Learning [J]. Computer Science, vol. 3942, 2006, pp. 124-131

[4] Manuel Mora, Rory O’Connor, Mahesh S. Raisinghani, An IT Service Engineering and Management Framework (ITS-EMF) [J], International Journal of Service Science, Management, Engineering, and Technology (IJSSMET).2011,2(2), pp.1-15

[5] Lu Han and Yi Ding, Development and Practice of Knowledge Service Platform Based on DSpace Advances in Computer Science and Education [C], 2012, Advances in Intelligent and Soft Computing, 2012, Volume 140, pp. 163-169

[6] Liang Xiao, Yanli Pei, A Task Context Aware Physical Distribution Knowledge Service System [J], International Journal of Knowledge and Systems Science (IJKSS), 2011, 2(1), pp. 17-31

[7] Herbert Lee, Keith Chan and Eric Tsui, Knowledge Mining Wikipedia: An Ontological Approach [J], International Journal of Knowledge and Systems Science (IJKSS), 2011, 2(1), pp. $50-60$

[8] Nicola Bicocchi, et al. Self-Organized Data Ecologies for Pervasive Situation-Aware Services: 
The Knowledge Networks Approach [J], IEEE Transactions on Systems, Man, and Cybernetics—part A: Systems and Humans, 2010, 40(4), pp. 789-802

[9] A Brogi, S Cortini, R Popescu, Composition-oriented Service Discovery [C], Proceedings of Software Composition, 2005, pp. 15-30

[10]. B Benatallab, M S Hacid, A Leger, C Rey, On Automating Web services Discovery [J]. International Journal on Very Large Data Bases, 2005, 14(1), pp. 84-96.

[11] H R Motahari Nezhad, G Y Xu and B Benatallah, Protocol-aware matching of web service interfaces for adapter development [C], Proceedings of the 19th international conference on World Wide Web, 2010, pp. 731-740.

[12] Hongxia Tong, Jian Cao, Shensheng Zhang and Minglu Li, A Distributed Algorithm for Web Service Composition Based on Service Agent Model [J], IEEE transactions on parallel and distributed system, 2011, 22(12), pp. 2008-2021 